\title{
Correction to: Automatic differentiation of Glaucoma visual field from non-glaucoma visual field using deep convolutional neural network
}

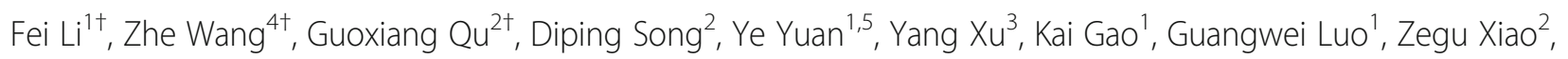
Dennis S. C. Lam ${ }^{5}$, Hua Zhong ${ }^{3^{*}}$, Yu Qiao ${ }^{2^{*}}$ and Xiulan Zhang ${ }^{1 *}$

\section{Correction to: BMC Med Imaging (2018) 18: 35 https://doi.org/10.1186/s12880-018-0273-5}

In the original version of this article [1], published on 4 October 2018, there was an error in the article title. The title of the original publication is: "Automatic differentiation of Glaucoma visual field from non-glaucoma visual filed using deep convolutional neural network", however, the correct title should be: "Automatic differentiation of Glaucoma visual field from non-glaucoma visual field using deep convolutional neural network", as presented in the title of this Correction article.

The same error appears in the Background section of the Abstract. "To develop a deep neural network able to differentiate glaucoma from non-glaucoma visual fields based on visual filed (VF) test results, we collected VF tests from 3 different ophthalmic centers in mainland China" should instead read: "To develop a deep neural network able to differentiate glaucoma from non-glaucoma visual fields based on visual field (VF) test results, we collected VF tests from 3 different ophthalmic centers in mainland China".

\section{Author details}

'Zhongshan Ophthalmic Center, State Key Laboratory of Ophthalmology, Sun Yat-sen University, Guangzhou, China. ${ }^{2}$ Guangdong key lab of Computer Vision \& Virtual Reality, Multimedia Research Center, Shenzhen Institutes of

\footnotetext{
*Correspondence: zhoculist@163.com; yu.qiao@siat.ac.cn; zhangx12@mail.sysu.edu.cn

${ }^{\dagger}$ Fei Li, Zhe Wang and Guoxiang Qu contributed equally to this work. ${ }^{3}$ Department of Ophthalmology, The First Affiliated Hospital of Kunming Medical University, Kunming, China

${ }^{2}$ Guangdong key lab of Computer Vision \& Virtual Reality, Multimedia Research Center, Shenzhen Institutes of Advanced Technology, Chinese Academy of Sciences, Shenzhen, China

'Zhongshan Ophthalmic Center, State Key Laboratory of Ophthalmology,

Sun Yat-sen University, Guangzhou, China

Full list of author information is available at the end of the article
}

Advanced Technology, Chinese Academy of Sciences, Shenzhen, China. ${ }^{3}$ Department of Ophthalmology, The First Affiliated Hospital of Kunming Medical University, Kunming, China. ${ }^{4}$ SenseTime Group Limited, Hong Kong, China. ${ }^{5}$ C-MER Dennis Lam Eye Hospital, Shenzhen, China.

Received: 7 May 2019 Accepted: 7 May 2019

Published online: 21 May 2019

\section{Reference}

1. Li F, Wang Z, Qu G, et al. Automatic differentiation of Glaucoma visual field from non-glaucoma visual filed using deep convolutional neural network. BMC Med Imaging. 2018;18(35) https://doi.org/10.1186/s12880-018-0273-5

(c) The Author(s). 2019 Open Access This article is distributed under the terms of the Creative Commons Attribution 4.0 International License (http://creativecommons.org/licenses/by/4.0/), which permits unrestricted use, distribution, and reproduction in any medium, provided you give appropriate credit to the original author(s) and the source, provide a link to the Creative Commons license, and indicate if changes were made. The Creative Commons Public Domain Dedication waiver (http://creativecommons.org/publicdomain/zero/1.0/) applies to the data made available in this article, unless otherwise stated. 